\title{
Device Classification-Based Context Management for Ubiquitous Computing using Machine Learning
}

\author{
Nalini A. Mhetre, Arvind V. Deshpande, Parikshit Narendra Mahalle
}

\begin{abstract}
Ubiquitous computing comprises scenarios where networks, devices within the network, and software components change frequently. Market demand and cost-effectiveness are forcing device manufacturers to introduce new-age devices. Also, the Internet of Things (IoT) is transitioning rapidly from the IoT to the Internet of Everything (IoE). Due to this enormous scale, effective management of these devices becomes vital to support trustworthy and high-quality applications. One of the key challenges of IoT device management is proactive device classification with the logically semantic type and using that as a parameter for device context management. This would enable smart security solutions. In this paper, a device classification approach is proposed for the context management of ubiquitous devices based on unsupervised machine learning. To classify unknown devices and to label them logically, a proactive device classification model is framed using a $k$-Means clustering algorithm. To group devices, it uses the information of network parameters such as Received Signal Strength Indicator (rssi), packet_size, number_of_nodes in the network, throughput, etc. Experimental analysis suggests that the well-formedness of clusters can be used to derive cluster labels as a logically semantic device type which would be a context for resource management and authorization of resources.
\end{abstract}

Keywords: Context Management, Device Classification, IoT Device Management, K-Means Clustering, Ubiquitous Computing, Unsupervised Machine Learning.

\section{INTRODUCTION}

$\mathrm{T}$ he term ubiquitous means "always existing everywhere" i.e., constantly available. In computer science, ubiquitous computing ('ubicomp') is a term where computing is accessible anytime and everywhere. Ubicomp can take place using any computing device, at any place, and in any format as opposed to desktop computing. Several ubicomp ideas have emerged in recent years which is nowadays termed as Internet of Things (IoT), a collection of smart, inter-connected objects using cutting edge internet technologies. Currently, the IoT is moving rapidly through various transformations and transitioning towards the Internet of Everything (IoE) [1]. A

Manuscript received on May 24, 2021

Revised Manuscript received on May 31, 2021.

Manuscript published on June 30, 2021.

* Correspondence Author

Nalini A. Mhetre*, Dept. of Computer Engg., Sinhgad College of Engg., Pune (Maharashtra), India. Email: nalini.mhetre@gmail.com

Arvind V. Deshpande, Dept. of Computer Engg., S.K.N. College of Engg., Pune (Maharashtra), India. Email: principal.skncoe@sinhgad.edu

Parikshit Narendra Mahalle, Dept. of Computer Engg., S.K.N. College of Engg., Pune (Maharashtra), India. Email: aalborg.pnm@gmail.com

(c) The Authors. Published by Blue Eyes Intelligence Engineering and Sciences Publication (BEIESP). This is an open access article under the CC BY-NC-ND license (http://creativecommons.org/licenses/by-nc-nd/4.0/) user interacts with the computer, which can exist in many different heterogeneous forms including laptops, computers, tablets, and everyday objects such as air conditioners, washing machines, stoves, fridges, TV, cars, even a pair of glasses, and several such physical objects (either smart or with an additional smart layer through various sensors). This rapid growth in devices and the availability of computing power to them leads to or demands context-aware and responsive application environments. Smart connectivity and context-aware computations are an important part of IoT [2] for various services.

The growth and spread estimate for the internet-connected devices is around 34 Billion [3] by the year 2020, which includes 20 Billion IoT devices. Reports also predict that IoT Device count will reach 76 Billion by the year 2025 [4], out of which 50 Billion will be connected to the internet. This explosion of devices [5] makes it crucial for systems to manage these devices to provide security and access control, which is an open issue [6]. One of the major challenges IoT faces is access control to its resources. Framing device-specific authorization policies would be difficult, time-consuming, and unscalable given a large number of heterogeneous IoT devices. Also, from a network security point of view, administrators may restrict the usage of certain types of devices. To address these problems, future solutions would require dealing with a group of devices rather than dealing with a single device. Thus, there is a need to classify devices having certain similarities. It would also be useful to automate the process of device grouping.

All major IoT devices or things in ubicomp are typically battery-operated or with limited energy. Also, the signal strength of the device has a significant impact on energy consumption and the communication it intends to do. Various computations, accessing resources, and more specifically the transmission of data consumes a lot of energy, and it is the main reason why energy consumption is one of the main constraints to consider when building ubicomp systems. Thus, to classify devices by taking energy and signal strength as primary parameters along with other networking parameters to study would be more useful from the context management perspectives. In recent times, the automatic classification of smart devices using different contexts including network packets [7], device ids [8] has been explored.

However, the majority of the work focuses on identifying devices based on their consistent features but not considering the changing state of the device during the lifetime of communication and resource utilization.

\section{Published By:}

Blue Eyes Intelligence Engineering and Sciences Publication (C) Copyright: All rights reserved.

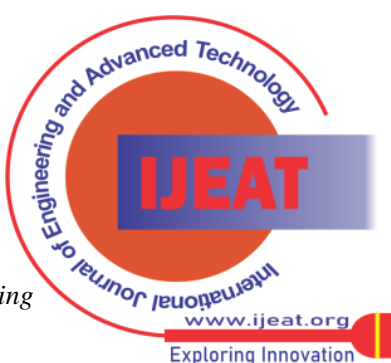


We have a different perspective in this paper: the purpose of device classification is to enable automated resource management. Hence, it is not required to detect the actual real-world type of a device. It is enough to consistently map devices to a theoretical/logical "device type" for which the system learns a specific set of access policies. Thus, manual labelling to the communication data records of real-world devices can be avoided. Basically, this abstract "device type" is going to represent one of the derived context for a device. The goal is to develop a technique to quickly and autonomously find the type of ubiquitous devices which should scale to a large number of existing devices and should also adapt to newly emerged devices. In this paper, a novel approach to classify devices using their networking features by applying unsupervised machine learning techniques is proposed. This model helps to find common patterns of network parameters in devices to group them under a particular class. A technique shows the usefulness of k-Means clustering algorithms in automating the process of device classification. using prominent network features such as the number_of_nodes, signal strength, energy consumption, throughput along with secondary features such as constant bit rate (cbrrate), packet size, overhead, delay and hop count for device grouping. We demonstrate the usefulness of our model to derive logically semantic context that could be used for resource management.

The rest of this paper is organized as follows. Section 2 focuses on motivation; Section 3 presents related work. The proposed approach and technical details are discussed in Section 4. The experimental results are given in section 5 . Finally, the paper is concluded in section 6.

\section{MOTIVATION}

We are living in an era of anything, anytime, anywhere paradigm. In the last two decades, advancements in technologies brought down hardware costs drastically and allowed device manufacturers to add communication technologies in tiny devices like heartbeat monitors to large appliances like TV, fridge, washing machine. More devices are being designed with Wi-Fi capabilities, and many heterogeneous devices are interconnected or connected in the sequel to allow resource sharing and communication with other network participating nodes. Technology coverage has shifted from traditional desktops to smart things [9]. Smart things observe, gather, and transmit data to offer personalized services. The contribution of the ubicomp system is critical to the deployment of personalized services in smart hyperspaces [9]. The IoT is about adding capabilities to these objects to connect them with the internet. An example of ubicomp is a smartwatch that alerts the user about a phone call and allows that call processing through that watch. Traditional methods of access control and security solutions may not be applicable as it is to this scenario. Historically, security requirements are considered to be relatively static because access control decisions do not change with context, nor do they account for changing environmental conditions. but, smart connectivity and context-aware computations are an important part of IoT [2] for various services to make them adaptive.

As we discussed previously, context plays a vital role in today's networks. Context-aware services respond and adapt to changes in their computing environment by designing policy rules [10]. As ubicomp comprises heterogeneous devices, context related to these devices [11] and their capabilities is important for context-aware applications . This context may affect the behavior of these applications as they not only use user interactions and their internal state information but also context information sensed during execution. The core issue of this problem is how to allocate or authorize the resources in the IoT system to accommodate the requirements imposed by applications. Due to the large scale and heterogeneity, long term context management of these devices becomes crucial. also, Resource-aware computing [12] is an approach to implement systems where the system continuously monitors the consumption of essential resources and can help the application make a decision based on resource availability now and in the future. These applications need to track existing resources, their capabilities [13], and their availability. For example, video streaming can be adjusted to the available bandwidth or signal and battery level, or the device may be asked to go to an area with better wireless local area network coverage [14].

The scope provided in the aforementioned points makes us look at device classification from the perspective of device context management. This essentially would be created through linking, monitoring, and analyzing some key parameters such as - signal strength, data packet size, energy consumption, transmission delay due to nodal hops, and the device and network-related features. Various factors affect the state of the network and device state. For Device-to-Device communication, in the context of IoT, there have been several approaches studied and validated. The Validity of such approaches and their respective feasibilities have also been tested successfully. These several approaches revolve around either Supervised or unsupervised environments or rule-based standard algorithms. After studying all these approaches, their respective strengths, and feasibility, we decided to take a novel approach of unsupervised machine learning algorithms based on clustering techniques to investigate unlabeled information of devices.

\section{RELATED WORK}

\section{A. Device Classification}

Classification of each device connected to a network and participating in communication is a tedious task, and there needs an approach to classify devices based on device capabilities. Significant efforts were made in the past by researchers to classify devices based on various device parameters or device contexts. Bharat [15] discussed classification based on features extracted from network traffic.

A. Sivanathan [16] worked on a multistage machine learning-based classification framework that uniquely identifies IoT devices with high accuracy.

Mahalle [17] discussed Decision theory-based Object Classification, and the paper has presented the logical framework for object classification to provide contextual information by considering energy parameters.

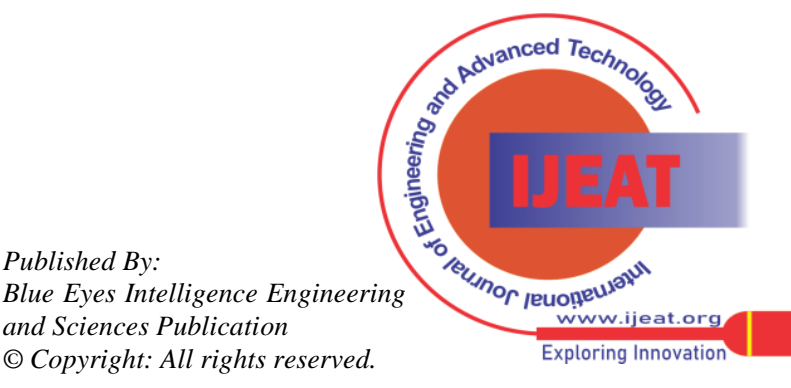


A. Sivanathan [18] presented a technique to classify IoT traffic based on network and device parameters like Sleep time, Active volume Avg. Packet size, Mean Rate, Peak / Mean rate Active time, No. of servers, No. of protocols, Unique DNS req., DNS interval NTP. Given the resulting traffic profile, the probability histogram of the sleep time attribute is studied and observed that there is a unique pattern for some IoT devices. This approach was to classify IoT and Non-IoT devices.

Y. Meidan [19] presented a machine learning approach for IoT device identification based on network traffic analysis. This work used supervised learning and trained a multistage classifier that can distinguish traffic generated by IoT and Non-IoT devices. S. Sharma [20] presented a generalized approach of incremental clustering to classify heterogeneous devices in a dynamic ubiquitous computing environment. The $\mathrm{HiCHO}$ technique is protocol neutral and based on attributes, and it's too general for dynamic dimensions. Kalmar [21] used the Hierarchical temporal memory (HTM) framework for context identification of objects facilitating context-aware services. Through different sets, this work proved that if the training data set was ideal and consistent; then the network could classify previously unseen context vectors related to objects quite efficiently.

S. V. Radhakrishnan [22] and A. J. Pinheiro [23] used network traffic analysis to classify devices with a non-machine learning approach. Earlier, M. Danieletto [24], addressed device classification based on ontology parameters while X. Feng [25] proposed a rule-based engine and P. R. J. Pego and L. Nunes [26] proposed approach-based custom communication properties to classify devices. Ke Gao [27] focused on the classification of only AP's using wavelet analysis of network packets. The overall perspective of the various researchers to classify devices is application specific.

Table I lists a summary of the related work for device classification based on various machine learning techniques.

\section{B. Gap Analysis}

Summary of related work shows that in the early days, the Machine Learning approach was not much used in the area of device classification. However, recent work employs machine learning techniques in this domain, particularly supervised. Recently, few attempts have been made to use unsupervised machine learning.

Most of the device classification models focus on packet data, while some consider very limited parameters

Table- I: Summary of related work

\begin{tabular}{|c|c|c|c|c|c|}
\hline $\begin{array}{c}\text { Existing } \\
\text { Work }\end{array}$ & Device classification parameters & ML & ML Type & Algorithm & Challenges \\
\hline [28] & $\begin{array}{c}\text { Source IP address, destination IP address, source and } \\
\text { destination port numbers, direction of the flow of traffic, } \\
\text { protocol used, number of packets transmitted, duration for } \\
\text { which the connection was made, and total data received in } \\
\text { bytes. }\end{array}$ & Yes & Supervised & KNN, NB, SVM, RF & Limited to Medical IOT devices \\
\hline [29] & Software and hardware specification & Yes & Supervised & KNN, NB, SVM, RF & Physical Attributes only \\
\hline [19] & Logical characteristics of the network traffic & Yes & Supervised & Multistage Classifier & $\begin{array}{l}\text { Two Parameters IOT and } \\
\text { Non-IoT Device }\end{array}$ \\
\hline [18] & $\begin{array}{c}\text { Sleep time, active volume, average packet size, mean rate, } \\
\text { peak to mean ratio, active time, number of servers, number } \\
\text { of protocols, unique DNS requests, DNS interval, NTP } \\
\text { interval, most frequent port number, and a label identifying } \\
\text { the IoT device }\end{array}$ & Yes & Unsupervised & k-Means & $\begin{array}{l}\text { Classification based on device } \\
\text { types. }\end{array}$ \\
\hline [30] & Device Events & Yes & Supervised & $\begin{array}{l}\text { KNN, Decision Tree, } \\
\text { Random Forest, SVM }\end{array}$ & Hypotheses testing based \\
\hline [16] & Packet-level and flow-level & Yes & Supervised & Multistage Classifier & Signal Strength Not considered \\
\hline [31] & Size of the first $\mathrm{N}$ packets sent and received, arrival times & Yes & Supervised & Random Forest classifier & Limited Dataset \\
\hline [7] & $\begin{array}{c}\text { time when the packet is sent out or received, packet length, } \\
\text { protocol, MAC address of source device and destination } \\
\text { device }\end{array}$ & Yes & Deep Learning & CNN & Training \\
\hline [32] & $\begin{array}{l}\text { UDP,NTP,SSDP data packets. Average packet size and } \\
\text { average rate attributes are used for clustering alogorithm. }\end{array}$ & Yes & Unsupervised & k-Means, PCA & $\begin{array}{l}\text { PCA is highly affected by } \\
\text { outliners. One model for one } \\
\text { type of device. }\end{array}$ \\
\hline [33] & Number of nodes, number of edges, & Yes & Unsupervised & Deep Learning & $\begin{array}{l}\text { ML used for network alignment } \\
\text { problem. }\end{array}$ \\
\hline [34] & Device Trafic (NTP, ARP, RTSP etc.) & Yes & Semi Supervised & ReliefF, KNN, PCA & $\begin{array}{l}\text { Unsupervised clustering method } \\
\text { is unclear. }\end{array}$ \\
\hline
\end{tabular}

For classification, like, energy or software/hardware specifications, or vendor information, etc. It is also observed that the data set used was diverse and specific to limited types of devices. The use of signal strength of devices along with other networking parameters while classifying them is unaddressed. The unstructured information makes unsupervised learning a suitable solution to identify patterns to form logical/theoretical groups of devices.

\section{PROPOSED WORK}

In ubiquitous computing environments, smart applications need methods for classifying devices based on certain criteria such as physical, logical, and networking attributes.

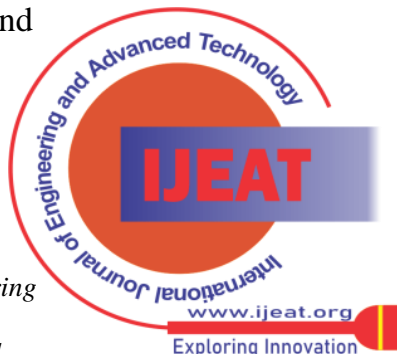


This classification may be helpful in applying rules, security policies, context management, and in in device administration. In this section, the proposed approach and methodology to classify devices is discussed. Further, an algorithm to categorize IoT devices using unsupervised machine learning is presented. An autonomous technique to classify IoT devices into different groups based on their network event information could be useful in context management of many applications

\section{A. Proposed Approach and Methodology}

The main modules of the proposed approach as following:

- Data acquisition

- Data Preprocessing and Feature Selection,

- Device Clustering

- Cluster Labelling

Fig.1 shows these modules and methodology of proposed work.

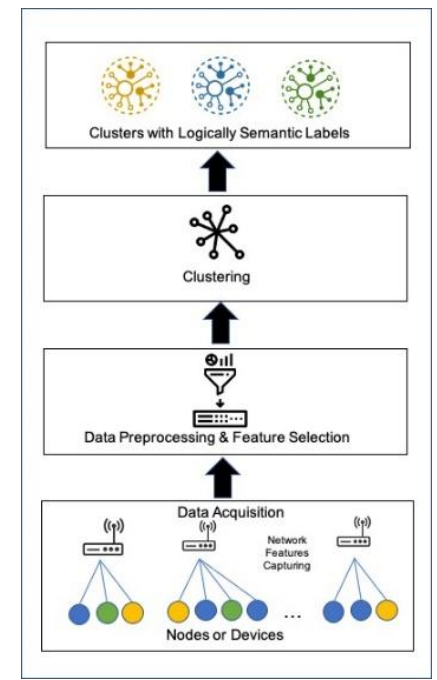

Fig. 1.Proposed approach and methodology

\section{i. Data Acquisition}

When IoT devices are connected to wireless networks, network event logs of these devices contain useful information of various features such as number_of_nodes in the network, rssi, packet_size, overhead, throughput, delay, energy_consumed, etc. We propose to use this information as a dataset to do clustering of the devices. This dataset could be represented as a collection of device event records as:

$\mathrm{D}=\{\mathrm{D} 1, \mathrm{D} 2, \ldots, \mathrm{Di}, \ldots, \mathrm{Dn}\}$

Where, Di represents a record of network event information of various features of the ith device. Each Di contains features information that corresponds to device $\mathrm{i}$ is given in eq. 2:

$\mathrm{Di}=\left\{\mathrm{n}_{\mathrm{i}}\right.$, rssi $_{\mathrm{i}}$, throughput $_{\mathrm{i}}$, energy $_{\mathrm{i}}, \ldots$, others $\left._{\mathrm{i}}\right\}$

Where, $n_{i}$ represents the number_of_nodes in the network when an ith device is connected. $\mathrm{rssi}_{\mathrm{i}}$ represents the Received Signal Strength Indicator of the ith device, throughput $t_{i}$ is the throughput of ith device, energy $y_{i}$ represent energy_consumed of ith device, ... and so on. All other features' that are not considered in this work is represented collectively as others ${ }_{i}$. The features that are actually considered for our work are given in experimentation section discussed further.

\section{ii. Data Preprocessing and Feature Selection}

In the data preprocessing module, values for the selected features are analyzed and normalized on a scale of $0-1$. Feature scaling with normalization is done in a dataset to bring the values of these parameters to a common scale, without changing differences in their ranges of values. It is required because ranges of feature values are varying. If not done, the feature with a higher value range starts dominating when calculating distances regardless of unit. So, to avoid algorithm to be biased towards features with higher value range normalization is done.

Further, correlation between selected features is identified to evaluate their importance in cluster formation. Dataset $\mathrm{D}$ is extracted for features of interest by filtering out othersi. It is represented as:

$\mathrm{D}=\left\{\mathrm{x} \mid \mathrm{x} \in \mathrm{Di}, \mathrm{x} \neq\right.$ others $\left._{\mathrm{i}}\right\}$ i.e. $\mathrm{D} \subset \mathrm{Di}$

We used Kendall rank correlation method to find out correlated features. This method measures strength of dependence between two variables. Features with high correlation creates redundant information, so, we selected only those with low correlation.

\section{iii. Device Clustering}

We aim to categorize IoT devices using selected features and classify them accordingly. For this, we need to identify certain unseen patterns amongst selected features. These patterns are to be used to form clusters of devices. Here, we propose a device classification model based on unsupervised machine learning and by using the k-Means clustering algorithm. The k-Means clustering algorithm is widely used unsupervised machine learning technique to make inferences from datasets without referring to known or labeled outcomes. It is an iterative method which uses a centroid per cluster. The dataset of selected features is represented by eq. 1 which is an input to the k-Means clustering algorithm. Here, k refers to a finite number of clusters to be formed.

Each Di gets assigned to one of the k clusters based on its minimum Euclidian distance from a particular centroid. At the end of each iteration of K-Means, each centroid is updated as an average distance of all records assigned to it. Algorithm ends when centroid values stop updating further. The output clusters refer to a group of devices that are formed due to certain similar patterns in selected features. Let, $\mathrm{C}$ be the set of $\mathrm{k}$ output clusters denoted as:

$\mathrm{C}=\{\mathrm{C} 1, \mathrm{C} 2, \ldots, \mathrm{Ck}\}$

Suppose $k=2$, then $C=\{C 1, C 2\}$ i.e. 2 clusters $C 1 \& C 2$ of devices will be formed and every device in dataset $\mathrm{D}$ is allocated to one of these 2 clusters as follows:

$$
\begin{aligned}
& C 1=\{d \mid d \in D, d \notin C 2\} \text { i.e. } C 1 \subset D \\
& C 2=\{d \mid d \in D, d \notin C 1\} \text { i.e. } C 2 \subset D \\
& C 1 \cap C 2=\varnothing \\
& C 1 \cup C 2=D
\end{aligned}
$$

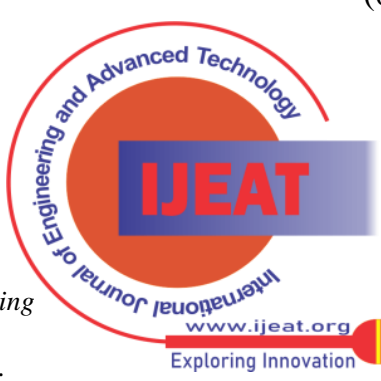


If the optimal value of the number of clusters is $\mathrm{k}$, then clusters $\mathrm{C} 1, \mathrm{C} 2, \ldots, \mathrm{Ck}$ will be formed. Accordingly, eq. 3 and eq. 4 can be extended as:

$$
\begin{aligned}
& (\mathrm{C} 1 \cap \mathrm{C} 2 \cap \ldots \cap \mathrm{Ck})=\emptyset \\
& (\mathrm{C} 1 \cup \mathrm{C} 2 \cup \ldots \cup \mathrm{Ck})=\mathrm{D}
\end{aligned}
$$

Cluster reformation is required periodically when dataset and correlation changes to accommodate new devices.

\section{iv. Cluster Labeling}

Each cluster formed is further analyzed by considering actual values of selected features from the dataset to derive semantic labels for it manually. These labels given to clusters represent the logical type of the device indicating a specific category. This categorization characterizes the classification of IoT devices as an outcome of our approach. These labels can be considered as context information of the device belonging to that category which may serve for policy enforcement while granting resources to them.

\section{B. Algorithms}

The algorithm AssignClusters() to classify devices based on their selected features using the clustering technique is summarized in pseudo-code as depicted in Algorithm 1.

\begin{tabular}{|l|}
\hline $\begin{array}{l}\text { Algorithm 1: AssignClusters() // Identify clusters and } \\
\text { assign devices to clusters }\end{array}$ \\
\hline Input: $\mathrm{D}[\mathrm{C}$ \\
Output: $\mathrm{C}[] \quad \mathrm{C} 1, \mathrm{C} 2, \ldots, \mathrm{Ck}$ k number of clusters of \\
devices) \\
Method: \\
1. $\mathrm{N}[] \leftarrow$ normalize(D[]) \\
2. $\mathrm{F}[] \leftarrow$ corr(N[]) \\
3. Initialize itr $\leftarrow 15$ \\
4. $\mathrm{k} \leftarrow$ FindOptimalK(N[], itr) \\
5. $\mathrm{C}[] \leftarrow$ k-Means(k, N[]) \\
6. $\mathrm{R}[] \leftarrow$ ReduceDimentionsT-SNE(D[], \\
C.labels) \\
7. Get a cluster label from R for each record in D.
\end{tabular}

Input to AssignClusters() algorithm is a dataset D. D is a collection of vectors of features characterizing devices. The first step of algorithm 1 normalizes the dataset $\mathrm{D}$ between 0 and 1 . The normalized dataset is denoted as $\mathrm{N}$ which contains values of features on the common scale of 0 to 1 . In the next step of the algorithm, the correlation between normalized features is calculated to find the features of interest. Features with maximum correlation are selected for analysis. This dataset is used as a training dataset for the clustering model. The next step is to find out the optimal number of clusters for a given training dataset. FindOptimalK() algorithm finds optimal value for $\mathrm{k}$ by plotting distortion for every iteration of itr. It is described in Algorithm 2. Further, k-Means() clustering algorithm generates $\mathrm{k}$ number of clusters using this optimal value of $k$. C represents a list of $k$ clusters where each record in $\mathrm{D}$ is assigned to one of the $\mathrm{k}$ clusters. As $\mathrm{D}$ is multi-dimensional data, to visualize the clusters formed, the t-Distributed Stochastic Neighbor Embedding (t-SNE) method is used to map the output cluster labels. It is a dimensionality reduction algorithm that plots high-dimensional data to two or more dimensions suitable for visualization. t-SNE would be a better option compared to
Principal Component analysis (PCA) because it reduces dimensions with non-linear relationships and handles outliers very well. $\mathrm{R}$ denotes $\mathrm{k}$ clusters of data points with reduced dimensions. Cluster id for each record representing a specific IoT device is obtained from R. Data of the records belonging to a specific cluster can further be analyzed to infer the semantic meaning of that cluster. As per the semantic meaning derived, clusters can be labeled logically which could be used as context for device management.

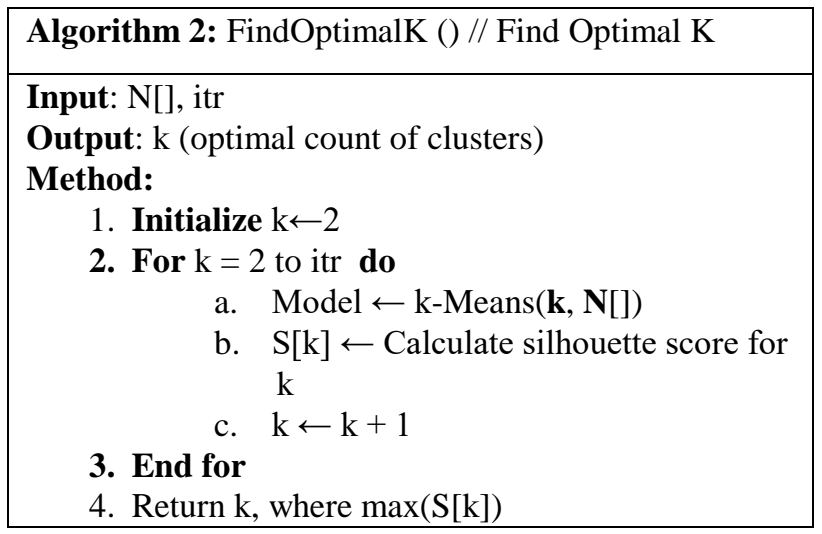

Algorithm 2 finds the optimal number of clusters for a given dataset. It assumes some finite value of itr. It iterates in a loop for values of $k$ from 1 to itr to generate the k-Means Model and to find silhouette score of the same. This algorithm returns the value of $\mathrm{k}$ where silhouette score is max.

\section{EXPERIMENTAL EVALUATION}

To evaluate our approach, we collected network event data of wireless nodes through simulation. Network simulator NS2 is used to perform the simulation.

In this section, we first provide an overview of the collected dataset and further discuss an experimental evaluation of the proposed methodology. Later, we also analyze the impact of different features in cluster formation

\section{A. Dataset}

Network event information of 221 wireless nodes was collected through several simulations of various wireless communication scenarios. The simulation setup of each scenario considered 802.11 mac layer protocol representing the IoT environment. Other input parameters to the simulation include the number_of_nodes, packet_size, signal strength indicator (ssi), cbrrate with varying values. The number of nodes considered for simulation range from 10 to 115 deployed in the area of $500 * 500$ meters.

The initial energy of each node set to 100 Jules. From the trace file generated through simulation, we extracted node-specific features such as total packet sent, total packets received, total packet dropped, total packet forwarded, packet delivery ratio, total hop count, overhead, throughput, delay, energy_consumed, residual energy, etc. Values of both input parameters and extracted features merged to form a dataset.

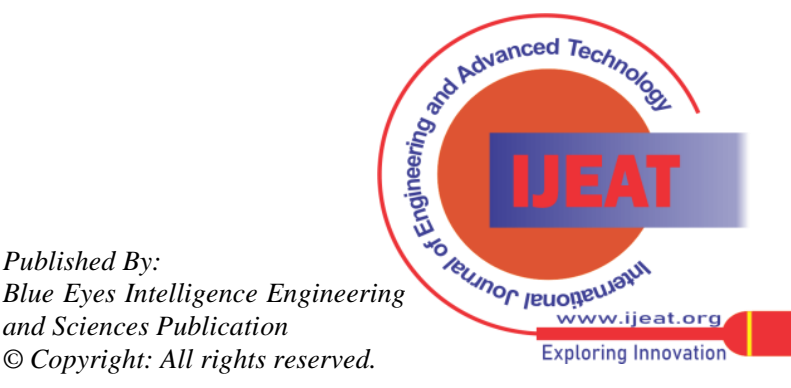




\section{B. Clustering}

A dataset comprising values of 21 features is generated through simulation trace file logs. However, this work considers a dataset of 9 selected features which includes the number_of_nodes in the network, ssi, packet_size, constant bit rate (cbrrate), average hop count, overhead, throughput, delay, energy_consumed. After cleaning and normalizing this data set, the correlation between these features is calculated. We used Kendall rank correlation method which measures non liner relationship between two variables. Table II shows the correlation matrix of these features.

Table II. Correlation of features of interest

\begin{tabular}{|c|c|c|c|c|c|c|c|c|c|}
\hline 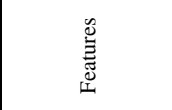 & 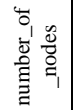 & $\bar{n}$ & 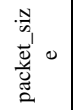 & $\begin{array}{l}\text { ָँّ } \\
\text { ปै }\end{array}$ & 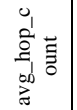 & 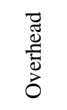 & 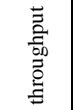 & $\frac{\pi}{d}$ & 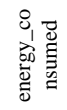 \\
\hline number_of_nodes & 1.000 & -0.145 & -0.333 & -0.342 & 0.032 & 0.532 & \begin{tabular}{|l|}
-0.129 \\
\end{tabular} & -0.063 & -0.252 \\
\hline ssi & -0.145 & 1.000 & 0.074 & 0.184 & -0.213 & 0.089 & 0.822 & 0.079 & 0.898 \\
\hline packet_size & -0.333 & 0.074 & 1.000 & 0.480 & -0.070 & -0.069 & 0.261 & 0.417 & 0.257 \\
\hline cbrrate & \begin{tabular}{|l|}
-0.342 \\
\end{tabular} & 0.184 & 0.480 & 1.000 & 0.059 & -0.122 & 0.264 & 0.387 & 0.324 \\
\hline avg_hop_count & 0.032 & -0.213 & -0.070 & 0.059 & 1.000 & -0.097 & -0.210 & 0.020 & -0.198 \\
\hline Overhead & 0.532 & 0.089 & \begin{tabular}{|l|}
-0.069 \\
\end{tabular} & -0.122 & -0.097 & 1.000 & -0.019 & -0.028 & 0.080 \\
\hline Throughput & -0.129 & 0.822 & 0.261 & 0.264 & -0.210 & -0.019 & 1.000 & 0.068 & 0.833 \\
\hline Delay & \begin{tabular}{|l|}
-0.063 \\
\end{tabular} & 0.079 & 0.417 & 0.387 & 0.020 & -0.028 & 0.068 & 1.000 & 0.223 \\
\hline $\begin{array}{c}\text { Energy } \\
\text { Consumed }\end{array}$ & -0.252 & 0.898 & 0.257 & 0.324 & -0.198 & 0.080 & 0.833 & 0.223 & 1.000 \\
\hline
\end{tabular}

Each cell in the table indicates the correlation coefficient value (in the range of -1 to 1 ) between two variables. These variables represent features and are shown in the respective row and column of the cell. The extreme correlation coefficient value -1 is a negative correlation and 1 is a positive correlation i.e., a perfectly linear relationship. Diagonal values show that each variable always perfectly correlates with itself and can be ignored as we want a correlation between different pairs of variables. Values closer to 1 and -1 indicate a strong nonlinear correlation. From Table II, it is observed that ssi, throughput, and energy_consumed are redundant as they are strongly correlated and are shown in darker cells. So, only ssi is considered.

\section{RESULT AND DISCUSSION}

\section{A. Cluster Validation}

The performance analysis of clusters formed is conducted by internal validation using the Silhouette technique. This is to get an appropriate value of $\mathrm{k}$ for which silhouette score is maximum. This method is considered robust as compared to other methods of finding k e.g. Elbow method. The silhouette score is a measure of the average similarity of the objects within a cluster and their distance to the other objects in the other clusters. Thus, it reflects both metrics of internal validation- Cohesion within each cluster, and Separation between different clusters. Fig.2. shows that for $k=3$ this score is maximum. It indicates that with current dataset, 3 optimal clusters can be formed. Also, this score is positive which indicates that there is proper cohesion and separation between clusters.

\section{B. Cluster Visualization}

Fig.3. depicts the output of the k-Means clustering algorithm implemented using the python toolkit. It shows three clusters generated and data points assigned to them. As the dataset is multidimensional, the t-SNE algorithm is used to visualize clusters in two dimensions. Data records of 221 devices are distributed as 71 in cluster ' 0 ', 65 in cluster ' 1 ', and 85 in cluster ' 2 '.

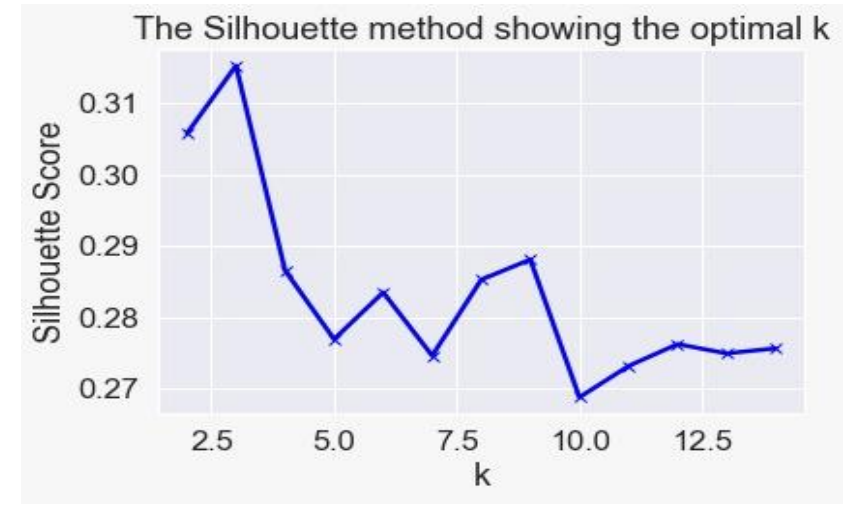

Fig. 2.Optimal k using Silhouette method

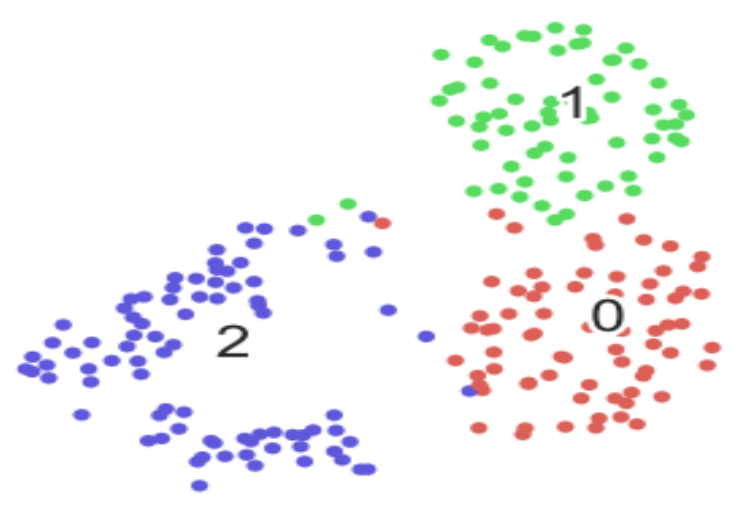

Fig. 3.Cluster visualization

\section{Cluster Analysis}

Internal evaluation of resultant clusters based on data itself suggests that clusters are well-formed. Figure 4 shows multivariate analysis in the form of a matrix of color scatter plots depicting the impact of selected features in cluster formation. It displays a distribution of values associated with specific features in resultant clusters. This involves checking out distributions as well as potential relationships and patterns amongst these features. Each data point is color-coded by the cluster to which it was assigned on the scale of normalized value. This helps in identifying which features give separation in the clusters and to observe each variable separately.

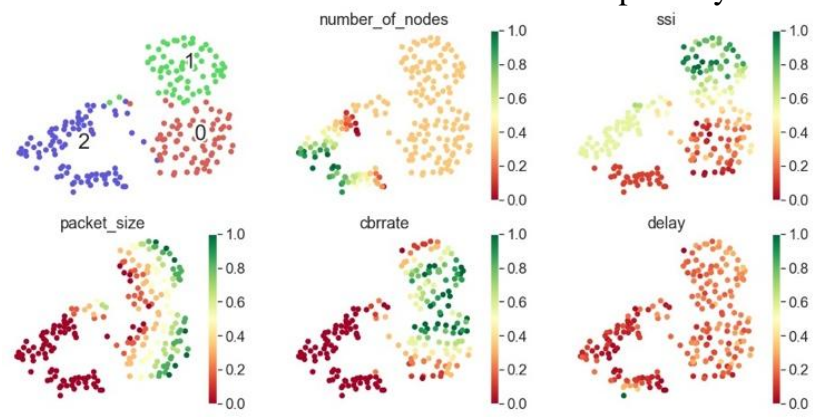

Fig. 4.Scatter plots for features distribution in clusters

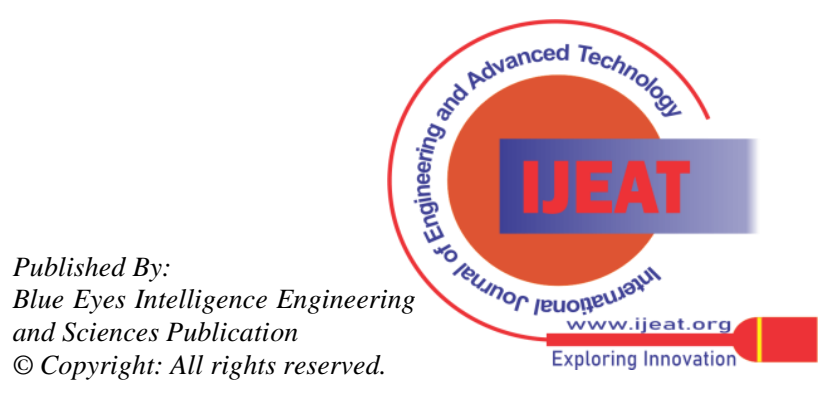


It can be observed that ssi majorly influences in clusters formation. Values of ssi in cluster 1 are high, in cluster 0 low, and cluster 2 are medium. Nodes with smaller values of packet_size, delay, and cbrrate has a strong impact in cluster 2. Cluster 0 and cluster 1 span the average number_of_nodes in the network with moderate delay. Extreme values of the number_of_nodes can be seen in cluster 2 with low delay. Cluster 2 has nodes with low cbrrate. Higher to medium-sized packet nodes are distributed in cluster 0 and cluster 1 . The summary of these observations is given in Table III.

Table III. Fuzzy analysis of node distribution in clusters

\begin{tabular}{|c|c|c|c|c|c|c|}
\hline & 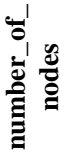 & 离 & 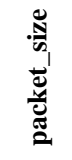 & 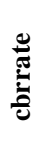 & $\frac{\vec{\pi}}{d}$ & 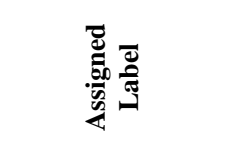 \\
\hline Cluster 0 & $\mathrm{M}$ & $\mathrm{L}$ & $\mathrm{M}, \mathrm{H}$ & $\mathrm{M}$ & $\mathrm{M}$ & CONSTRAINED \\
\hline Cluster 1 & $\mathrm{M}$ & $\mathrm{H}$ & $\mathrm{M}, \mathrm{H}$ & $\mathrm{H}$ & $\mathrm{M}$ & POWERFUL \\
\hline Cluster 2 & $\mathrm{~L}, \mathrm{H}$ & $\mathrm{M}$ & $\mathrm{L}$ & $\mathrm{L}$ & $\mathrm{L}$ & SEMI-POWERFUL \\
\hline
\end{tabular}

L: Low, M: Medium, H: High

This paper uses a fuzzy[35] approach for analyzing clusters. In an uncertain environment like IoT, resource owners cannot have crisp values of the assets. Therefore, fuzzy is more appropriate. Table III shows a Fuzzy analysis of node distribution in the clusters formed. Cluster inference by considering impact features is given below in the form of Mamdani fuzzy rules[36]:

Rule 1: IF ssi is L AND cbrrate is M AND delay is M THEN Cluster_label is CONSTRAINED

Rule 2: IF ssi is H AND cbrrate is H AND delay is M THEN Cluster_label is POWERFUL

Rule 3: IF ssi is M AND cbrrate is L AND delay is L AND packet_size is L THEN Cluster_label is SEMI-POWERFUL

Thus, Cluster 0 is labeled as CONSTRAINED, Cluster 1 is labeled as POWERFUL, and Cluster 2 is labeled as SEMI-POWERFUL. SEMI-POWERFUL cluster is characterized by smaller packet size and cbrrate.

\section{CONCLUSION}

In this paper, we propose a model to use networking information of IoT devices to classify them proactively using unsupervised machine learning. The k-Means clustering algorithm is used to identify groups of IoT devices using simulation data in wireless environments. As these parameters can be readily available within an organization, apparently our model can be used to facilitate a more intelligent IoT network. It will help organizations to design adaptive policies framework based on contextual information extracted from network parameters. Experimental results shows that the clusters are well formed. Logical labels given to these clusters further may help in classification of the similar devices proactively. Our work effectively shows the possibility to classify IoT devices and derive semantic context in autonomous way. In the future, we plan to explore the utilization of our device classification method in the area of adaptive access control and resource management. This model can further be extended for a dataset of new devices and real word dataset.

\section{REFERENCES}

1. D. Sudharshan, "Internet of Everything (IoE)," in Marketing in Customer Technology Environments, Emerald Publishing Limited, 2020, pp. 161-208.

2. "IoT Ecosystem - Internet of Things Forecasts \& Business Opportunities - Business Insider."

https://www.businessinsider.com/iot-ecosystem-internet-of-things-fo recasts-and-business-opportunities-2016-4-28?IR=T (accessed Jan. 14, 2020).

3. NelsonHilliard.com, "Managing the Explosion of Internet of Things (IoT) Data," www.nelsonhilliard.com.

https://www.nelsonhilliard.com/explosion-of-internet-of-things-iot-d ata/ (accessed Jan. 15, 2020).

4. Statista, "Internet of Things - number of connected devices worldwide 2015-2025," www.statista.com.

https://www.statista.com/statistics/471264/iot-number-of-connecteddevices-worldwide/.

5. S. Ornes, "The internet of things and the explosion of interconnectivity," Proc. Natl. Acad. Sci. U. S. A., vol. 113, no. 40, pp. 11059-11060, 2016, doi: 10.1073/pnas.1613921113.

6. www.fortinet.com, "Understanding the Iot Explosion and Its impact on enterprise security." [Online]. Available:

https://www.fortinet.com/content/dam/fortinet/assets/white-papers/ WP-Understanding-The-IoT-Explosion-And-Its-Impact-On-Enterpri se-Security.pdf.

7. L. Bai, L. Yao, S. S. Kanhere, X. Wang, and Z. Yang, "Automatic Device Classification from Network Traffic Streams of Internet of Things," Proc. - Conf. Local Comput. Networks, LCN, vol. 2018-Octob, pp. 597-605, 2019, doi: 10.1109/LCN.2018.8638232.

8. A. WG03, "AIOTI ALLIANCE FOR INTERNET OF THINGS INNOVATION Identifiers in Internet of Things (IoT), 2018.

9. J. Ma, L. T. Yang, B. O. Apduhan, R. Huang, L. Barolli, and M. Takizawa, "Towards a smart world and ubiquitous intelligence: A walkthrough from smart things to smart hyperspaces and UbicKids,' Int. J. Pervasive Comput. Commun., vol. 1, no. 1, pp. 53-68, 2005, doi: 10.1108/17427370580000113.

10. M. A. El Khaddar, M. Chraibi, H. Harroud, M. Boulmalf, M. Elkoutbi, and A. Maach, "A policy-based middleware for context-aware pervasive computing," Int. J. Pervasive Comput. Commun., vol. 11, no. 1, pp. 43-68, Apr. 2015, doi: 10.1108/IJPCC-07-2014-0039.

11. R. van Eijk, A. Salden, J. de Heer, A. Peddemors, P. Määttä, and V. Haataja, "Handling heterogeneity in context aware services," Int. J. Pervasive Comput. Commun., vol. 1, no. 1, pp. 25-30, Feb. 2005, doi: 10.1108/17427370580000110.

12. K. John, Ubiquitous Computing Fundamentals, vol. 53, no. 5. 2010.

13. B. Anggorojati, P. N. Mahalle, N. R. Prasad, and R. Prasad, "Capability-based access control delegation model on the federated IoT network," Int. Symp. Wirel. Pers. Multimed. Commun. WPMC, pp. 604-608, 2012.

14. D. Garlan, D. P. Siewiorek, A. Smailagic, and P. Steenkiste, "Project Aura: toward distraction-free pervasive computing," IEEE Pervasive Comput., vol. 1, no. 2, pp. 22-31, Apr. 2002, doi 10.1109/MPRV.2002.1012334.

15. B. A. Desai, D. M. Divakaran, I. Nevat, G. W. Peter, and M. Gurusamy, "A feature-ranking framework for IoT device classification," in $201911^{\text {th }}$ International Conference on Communication Systems \& Networks (COMSNETS), Jan. 2019, pp. 64-71, doi: 10.1109/COMSNETS.2019.8711210.

16. A. Sivanathan et al., "Classifying IoT Devices in Smart Environments Using Network Traffic Characteristics," IEEE Trans. Mob. Comput., vol. 18, no. 8, pp. 1745-1759, 2019, doi 10.1109/TMC.2018.2866249.

17. P. N. Mahalle, N. Rashmi Prasad, and R. Prasad, "Object Classification based Context Management for Identity Management in Internet of Things," Int. J. Comput. Appl., vol. 63, no. 12, pp. 1-6, Feb. 2013, doi: 10.5120/10515-5486.

18. A. Sivanathan et al., "Characterizing and classifying IoT traffic in smart cities and campuses," 2017 IEEE Conf. Comput. Commun. Work. INFOCOM WKSHPS 2017, pp. 559-564, 2017, doi: 10.1109/INFCOMW.2017.8116438.

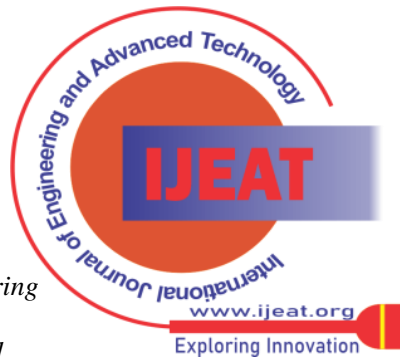


19. Y. Meidan et al., "ProfilloT: A machine learning approach for IoT device identification based on network traffic analysis," 2017, doi: 10.1145/3019612.3019878.

20. S. Sharma, S. Kapoor, B. R. Srinivasan, and M. S. Narula, "HiCHO : Attributes based Classification of Ubiquitous Devices," no. i, pp. 113-125, 2012.

21. A. Kalmar and R. Vida, "Towards context-aware mobile services through the use of Hierarchical Temporal Memory," in $201321^{\text {st }}$ International Conference on Software, Telecommunications and Computer Networks - (SoftCOM 2013), Sep. 2013, pp. 1-5, doi: 10.1109/SoftCOM.2013.6671905.

22. S. V. Radhakrishnan, A. S. Uluagac, and R. Beyah, "GTID: A Technique for Physical Device and Device Type Fingerprinting," IEEE Trans. Dependable Secur. Comput., vol. 12, no. 5, pp. 519-532, Sep. 2015, doi: 10.1109/TDSC.2014.2369033.

23. A. Aksoy and M. H. Gunes, "Automated IoT Device Identification using Network Traffic," in ICC 2019 - 2019 IEEE International Conference on Communications (ICC), May 2019, pp. 1-7, doi: 10.1109/ICC.2019.8761559.

24. M. Danieletto, N. Bui, and M. Zorzi, “An ontology-based framework for autonomic classification in the Internet of things," IEEE Int. Conf. Commun., no. July, 2011, doi: 10.1109/iccw.2011.5963599.

25. X. Feng, Q. Li, H. Wang, and L. Sun, "Acquisitional Rule-based Engine for Discovering Internet-of-Things Devices," Usenix'18, 2018.

26. P. R. J. Pego and L. Nunes, "Automatic discovery and classifications of IoT devices," Iber. Conf. Inf. Syst. Technol. Cist., 2017, doi: 10.23919/CISTI.2017.7975691.

27. Ke Gao, C. Corbett, and R. Beyah, "A passive approach to wireless device fingerprinting," in 2010 IEEE/IFIP International Conference on Dependable Systems \& Networks (DSN), Jun. 2010, pp. 383-392, doi: 10.1109/DSN.2010.5544294.

28. D. Arora, K. F. Li, and A. Loffler, "Big data analytics for classification of network enabled devices," Proc. - IEEE $30^{\text {th }}$ Int. Conf. Adv. Inf. Netw. Appl. Work. WAINA 2016, pp. 708-713, 2016, doi: 10.1109/WAINA.2016.131.

29. A. Mavrogiorgou, A. Kiourtis, and D. Kyriazis, "A Comparative Study of Classification Techniques for Managing IoT Devices of Common Specifications," vol. 3, no. January 2019, 2017, pp. 67-77.

30. A. J. Pinheiro, J. de M. Bezerra, C. A. P. Burgardt, and D. R. Campelo, "Identifying IoT devices and events based on packet length from encrypted traffic," Comput. Commun., 2019, doi: 10.1016/j.comcom.2019.05.012.

31. M. Shahid et al., "IoT Devices Recognition Through Network Traffic Analysis," 2018 IEEE Int. Conf. Big Data (Big Data), no. 978-1-5386-5035-6, 2019, doi: 10.1109/BigData.2018.8622243.

32. A. Sivanathan, H. H. Gharakheili, and V. Sivaraman, "Detecting Behavioral Change of IoT Devices Using Clustering-Based Network Traffic Modeling," IEEE Internet Things J., vol. 7, no. 8, pp. 7295-7309, 2020, doi: 10.1109/JIOT.2020.2984030.

33. D. Zhu, Y. Sun, H. Du, N. Cao, T. Baker, and G. Srivastava, "HUNA: A Method of Hierarchical Unsupervised Network Alignment for IoT," IEEE Internet Things J., vol. 8, no. 5, pp. 3201-3210, 2021, doi: 10.1109/JIOT.2020.3020951.

34. S. Marchal, M. Miettinen, T. D. Nguyen, A.-R. R. Sadeghi, and N. Asokan, "AuDI: Toward Autonomous IoT Device-Type Identification Using Periodic Communication," IEEE J. Sel. Areas Commun., vol. 37, no. 6, pp. 1402-1412, Jun. 2019, doi: 10.1109/JSAC.2019.2904364.

35. L. A. Zadeh, "Fuzzy sets," Inf. Control, vol. 8, no. 3, pp. 338-353, Jun. 1965, doi: 10.1016/S0019-9958(65)90241-X.

36. E. H. Mamdani and S. Assilian, "An experiment in linguistic synthesis with a fuzzy logic controller,” Int. J. Man. Mach. Stud., vol. 7, no. 1, pp. 1-13, 1975, doi: 10.1016/S0020-7373(75)80002-2.

\section{AUTHORS PROFILE}

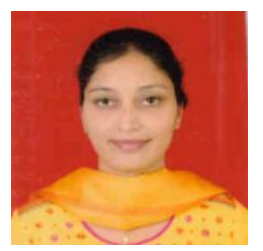

Ms. Nalini A. Mhetre, is currently working as an Assistant Professor in Department of Computer Engineering at STES's Sinhgad College of Engineering, Pune, India. She has obtained B.E. in Computer Engineering from Shivaji University, Kolhapur, India and M.E. in Computer Science and Engineering from University of Pune, Pune, India. Currently, she is pursuing Ph.D. in Computer engineering from Savitribai Phule Pune University, Pune, India. She has more than 19 years of teaching and research experience. She has contributed to several research publications at various national and international journals and conferences. Her recent research interests include Machine Learning, Data Science, Internet of Things (IoT), Identity Management, and Security.

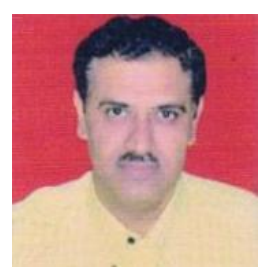

Dr. Arvind V. Deshpande, is the Principal at STES's Smt. Kashibai Navale College of Engineering, Pune, India. He has more than 28 years of teaching and research experience. He is a member of Board of Studies in Computer Engineering, Savitribai Phule Pune University, Pune, India. He is life member of ISTE. He has also remained technical program committee member for International Conferences and Symposium. He has contributed in several research publications at various national and international journals and conferences. His research area includes Image Processing, Internet of Things and Security

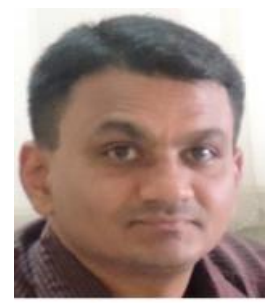

Dr. Parikshit Narendra Mahalle, has obtained his B.E in Computer Science and Engineering from Sant Gadge Baba Amravati University, Amravati, India and M.E. in Computer Engineering from University of Pune, Pune, India. He completed his Ph.D. in Computer Science and Engineering specialization in Wireless Communication from Aalborg University, Aalborg, Denmark. He is PostDoc Researcher at CMI, Aalborg University, Copenhagen, Denmark. He has more than 19 years of teaching and research experience. He has been a member board of studies in computer engineering, Savitribai Phule Pune University (SPPU), Pune, India. He has been a member - Board of studies in computer engineering, SPPU. He is member - BoS coordination committee in computer engineering, SPPU. He is also serving as member- Technical committee, SPPU. He is IEEE member, ACM member, Life member CSI and Life member ISTE. He is paper reviewer for Springer journal of Wireless Personal Communications and Elsevier journal of Applied Computing and Informatics. He has also remained technical program committee member for International conferences and symposium like IEEE ICC - 2014, IEEE ICACCI 2013, IEEE ICC 2015 - SAC-Communication for Smart Grid, IEEE ICC 2015 - SAC-Social Networking, IEEE ICC 2014 - Selected Areas in Communication Symposium, IEEE INDICON 2014, CSI ACC 2014, IEEE GCWSN 2014, GWS 2015, GLOBECOMM 2015. Currently he is working as Professor and Head in Department of Computer Engineering at STES's Smt. Kashibai Navale College of Engineering, Pune, India.
Published By: Blue Eyes Intelligence Engineering and Sciences Publication (C) Copyright: All rights reserved.

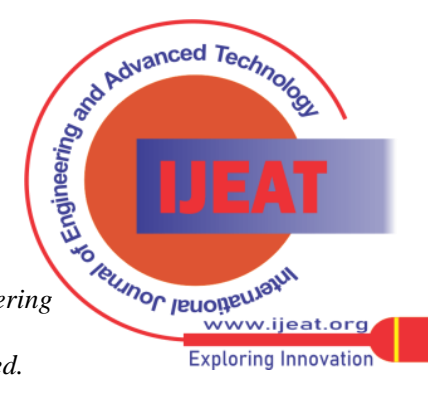

